\title{
Molnupiravir: First Approval
}

\author{
Yahiya Y. Syed ${ }^{1}$
}

Published online: 20 February 2022

(c) Springer Nature Switzerland AG 2022

\begin{abstract}
Molnupiravir (Lagevrio ${ }^{\circledR}$ ) is an orally-administered antiviral prodrug that inhibits replication of RNA viruses through viral error induction. It is being developed by Merck and Ridgeback Biotherapeutics for the prevention and treatment of Coronavirus disease 2019 (COVID-19). Molnupiravir received its first approval on 4 November 2021 in the UK for the treatment of mild to moderate COVID-19 in adults with a positive severe acute respiratory syndrome coronavirus 2 (SARS-CoV-2) diagnostic test and who have at least one risk factor for developing severe illness. Molnupiravir is filed for approval and has emergency use authorization for the treatment of COVID-19 in several countries, including the USA, Japan and those in the EU. This article summarizes the milestones in the development of molnupiravir leading to this first approval for COVID-19.
\end{abstract}

\section{Digital Features for this AdisInsight Report can be found at https://doi.org/10.6084/m9.figshare.19142618}

\section{Molnupiravir (Lagevrio $\left.{ }^{\oplus}\right)$ Key Points}

A broad-spectrum RNA antiviral prodrug being developed by Merck and Ridgeback Biotherapeutics for the treatment of COVID-19

Received its first approval on 4 November 2021 in the UK

Approved for use in adults with mild to moderate COVID-19 at risk of progression to severe illness

\section{Introduction}

Molnupiravir (Lagevrio ${ }^{\circledR}$ ) is an orally-administered, smallmolecule, antiviral prodrug that inhibits replication of RNA viruses through viral error induction [1]. The drug was

This profile has been extracted and modified from the AdisInsight database. AdisInsight tracks drug development worldwide through the entire development process, from discovery, through preclinical and clinical studies to market launch and beyond.

Yahiya Y. Syed

dru@adis.com

1 Springer Nature, Mairangi Bay, Private Bag 65901, Auckland 0754, New Zealand invented by Drug Innovation Ventures at Emory (DRIVE; wholly owned by Emory University) and is being developed by Merck, in collaboration with Ridgeback Biotherapeutics, for the prevention and treatment of Coronavirus disease 2019 (COVID-19) [1, 2]. Severe acute respiratory syndrome coronavirus 2 (SARS-CoV-2), the causative virus of COVID-19, uses an RNA-dependent RNA polymerase (RdRp) complex for replication [3]. Therefore, RdRp is a promising target for both developing new antiviral drugs against SARS-CoV-2 as well as for repurposing existing ones [4]. A number of drug candidates with activity against SARS-CoV-2 RdRp are being evaluated for the treatment of COVID-19 [4, 5]. Remdesivir, an intravenously administered RdRp inhibitor, is approved for the treatment of adults and paediatric patients with COVID-19 [6]. However, new treatment options are required to prevent disease progression in at-risk populations; in addition to improving patient-level clinical outcomes, such treatments can reduce healthcare system burden. In treating COVID-19, oral pills are preferable to intravenous drugs in terms of patient convenience, use during self-isolation, faster treatment uptake, healthcare resource use, scalability, logistics and cost.

Molnupiravir received its first approval on 4 November 2021 in the UK for the treatment of mild-to-moderate COVID-19 in adults with a positive SARS-CoV-2 diagnostic test and who have at least one risk factor for developing severe illness $[7,8]$. The recommended dosage of molnupiravir is $800 \mathrm{mg}$ every $12 \mathrm{~h}$ for 5 days. It should be administered as soon as possible after a COVID-19 diagnosis and within 5 days of symptom onset [7]. Molnupiravir underwent an accelerated first-in-human clinical trial programme through 


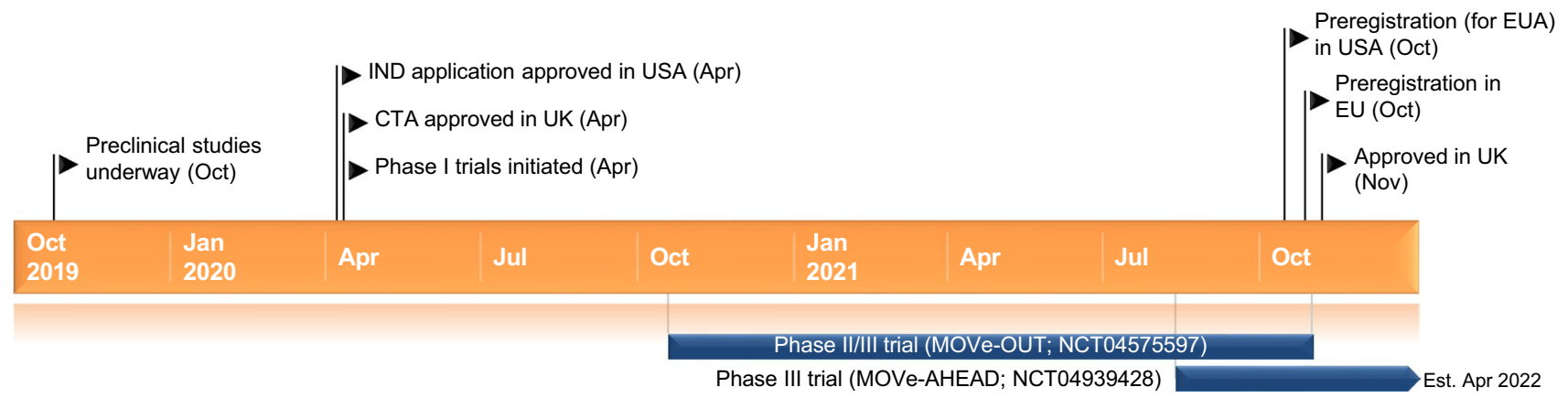

Key milestones in the development of molnupiravir for COVID-19. CTA clinical trial application, EUA emergency use authorization, IND investigational new drug

extraordinary collaboration between the drug developers, contract research organization and regulatory authorities [2].

Molnupiravir has been filed for approval and has emergency use authorization for the treatment of COVID-19 in several countries, including the USA, Japan and those in the EU. In addition, a multinational phase III trial is evaluating molnupiravir for post-exposure prophylaxis against COVID-19.

\subsection{Company Agreements}

In May 2020, Ridgeback Biotherapeutics entered into a licensing agreement with Merck for the development of molnupiravir. Under the terms of agreement, Merck has acquired exclusive worldwide rights to develop and commercialise molnupiravir and related molecules $[9,10]$. Ridgeback Biotherapeutics acquired an exclusive license to DRIVE's molnupiravir in March 2020 [11].

In June 2021, Cipla, Dr Reddys Laboratories, Emcure Pharmaceuticals Limited, Sun Pharmaceutical Industries and Torrent Pharmaceuticals entered into a collaboration agreement to conduct a clinical trial of molnupiravir in the treatment of mild COVID-19 in an outpatient setting in India. In March and April 2021, the five companies had individually entered into a non-exclusive voluntary licensing agreement with Merck to manufacture and supply molnupiravir to India and over 100 low- and middle-income countries [12]. As of January 2022, Merck has signed nonexclusive licensing agreements with additional generic drug manufactures in India.
In October 2021, Merck granted a license to the Medicines Patent Pool to increase broad access to molnupiravir in low- and middle-income countries upon regulatory approvals. Under this agreement, Merck, Ridgeback Biotherapeutics and Emory University will not receive royalties for molnupiravir sales as long as COVID-19 remains classified as a Public Health Emergency of International Concern by the World Health Organization [13].

In January 2022, Merck and Ridgeback Biotherapeutics entered into a long-term supply agreement with UNICEF, under which Merck will allocate up to 3 million courses of molnupiravir to UNICEF during the first half of 2022 for distribution in more than 100 low- and middle-income countries upon local regulatory authorizations [14]. Merck also has a procurement agreement with the US Government, under which the US Government purchased a total of approximately 3.1 million courses of molnupiravir upon emergency use authorization by the US FDA [14].

\section{Scientific Summary}

\subsection{Pharmacodynamics}

Molnupiravir is an orally available prodrug that is rapidly converted in plasma by host's esterases to the ribonucleoside analogue N-hydroxycytidine (NHC) [15]. After intracellular uptake, NHC is phosphorylated into its pharmacologically active ribonucleoside triphosphate

Chemical structure of molnupiravir<smiles>CC(C)C(=O)OC[C@H]1O[C@@H](n2ccc(=NO)[nH]c2=O)[C@H](O)[C@@H]1O</smiles> 
(NHC-TP). NHC-TP is incorporated into viral RNA by the RdRp, resulting in viral error induction (i.e. accumulation of errors in the viral genome ultimately leading to inhibition of replication) [7, 16, 17]. Consistent with this mechanism of action, molnupiravir treatment was associated with numerically higher SARS-CoV-2 viral RNA error rates versus placebo in patients with COVID-19 in clinical trials [18-20].

Molnupiravir inhibited SARS-CoV-2 in cell culture assays, with $50 \%$ effective concentrations $\left(\mathrm{EC}_{50}\right)$ ranging between 0.32 and $2.66 \mu \mathrm{M}$ [7]. Molnupiravir has broad-spectrum in vitro activity against SARS-CoV-2 variants of concern such as B1.1.529 (omicron) [21-26], and B.1.1.7 (alpha), B.1351 (beta), P.1 (gamma) and B.1.617.2 (delta) [7]. The $\mathrm{EC}_{50}$ values were 1.86-1.95 $\mu \mathrm{M}$ [21], and 1.59, 1.77, 1.32 and $1.68 \mu \mathrm{M}$ [7], respectively. In immunodeficient mice implanted with human lung tissue, therapeutic and prophylactic administration of molnupiravir inhibited SARS-CoV-2 replication in vivo [27]. Treatment of SARS-CoV-2-infected ferrets with molnupiravir reduced SARS-CoV-2 levels in the upper respiratory tract and completely blocked transmission of the virus to untreated contact animals [28]. Molnupiravir inhibited SARS-CoV-2 in human airway epithelial cell cultures; in SARS-CoV-2-infected mice, molnupiravir decreased viral load and body weight loss and improved pulmonary function [29].

The potential for SARS-CoV-2 resistance to molnupiravir is low [30]. In phase II trials of molnupiravir in patients with COVID-19, no amino acid substitutions in SARS-CoV-2 associated with resistance to NHC have been detected [7]. Preclinical data suggest that molnupiravir is of low risk for genotoxicity or mutagenicity in clinical use [31-33].

\subsection{Pharmacokinetics}

The pharmacokinetics of molnupiravir have been evaluated in heathy volunteers [15] and patients with COVID19 [19, 34]. According to a population pharmacokinetic model, plasma NHC pharmacokinetics following single and multiple twice-daily molnupiravir administrations can be adequately described by a linear two-compartment model, with sigmoid absorption and first-order elimination [35]. Molnupiravir pharmacokinetics did not differ significantly between healthy volunteers and COVID-19 patients [35]. In patients with COVID-19, NHC exhibited doseproportional pharmacokinetics and the exposures were comparable across all evaluated demographic and clinical characteristics, including age, sex, body-mass index, body weight, ethnicity and kidney function [36].

Following oral administration of molnupiravir $800 \mathrm{mg}$ every $12 \mathrm{~h}$, the median time to peak plasma NHC concentrations was $1.5 \mathrm{~h}$ [7]. In healthy volunteers receiving a single molnupiravir $200 \mathrm{mg}$ dose with a high-fat meal, NHC peak concentration was reduced by $35 \%$, while area under the plasma concentration was not significantly affected. NHC does not bind to plasma protein and it has an effective half-life of $\approx 3.3 \mathrm{~h}$. In healthy volunteers, $\leq 3 \%$ of the administered dose is excreted as NHC in the urine [7].

\subsection{Therapeutic Trials}

\subsubsection{Non-hospitalized Patients}

Molnupiravir was evaluated independently in outpatients with PCR-confirmed SARS-CoV-2 infection in a phase Ib/IIa trial (NCT04746183), using the AGILE platform. From the

\section{Features and properties of molnupiravir}

\begin{tabular}{|c|c|}
\hline Alternative names & Lagevrio; EIDD-2801; MK-4482 \\
\hline Class & Antivirals; esters; hydroxylamines; pyrimidinones; ribonucleosides; small molecules \\
\hline Mechanism of action & Viral replication inhibitors \\
\hline Route of administration & Oral \\
\hline Pharmacodynamics & $\begin{array}{l}\text { Molnupiravir is hydrolysed to N-hydroxycytidine }(\mathrm{NHC}) \text { which is phosphorylated to pharmacologically active } \\
\text { N-hydroxycytidine triphosphate. Inhibits SARS-CoV-2 replication via viral error induction; robust in vitro and } \\
\text { in vivo activity against SARS-CoV-2; retains activity against SARS-CoV-2 variants }\end{array}$ \\
\hline Pharmacokinetics of NHC & $\begin{array}{l}\mathrm{t}_{\max } 1.5 \mathrm{~h} \text {; a high-fat meal reduces } \mathrm{C}_{\max } \text { by } 35 \% \text {; does not bind to plasma proteins; } \mathrm{t}_{1 / 2} 3.3 \mathrm{~h} \text {; gender, race, age, and } \\
\text { kidney and liver impairment have no clinically relevant effect }\end{array}$ \\
\hline Most frequent adverse events & Diarrhoea, nausea, dizziness, headache \\
\hline \multicolumn{2}{|l|}{ ATC codes } \\
\hline WHO ATC code & J05A-X (other antivirals) \\
\hline EphMRA ATC code & J5B9 (antivirals, others) \\
\hline Chemical name & $\begin{array}{l}\text { [(2R,3S,4R,5R)-3,4-dihydroxy-5-[4-(hydroxyamino)-2-oxopyrimidin-1-yl]oxolan-2-yl]methyl 2-methylpro- } \\
\text { panoate }\end{array}$ \\
\hline
\end{tabular}


dose-escalating phase Ib component, a dosage of $800 \mathrm{mg}$ twice daily for 5 days was selected for the phase IIa component [34].

Molnupiravir significantly reduced nasopharyngeal SARS-CoV-2 load in outpatients with COVID-19 in a randomized, double-blind, placebo-controlled phase II trial (NCT04405570) [20]. Patients with confirmed SARS-CoV-2 infection and symptom onset within 7 days were randomized to molnupiravir 200,400 or $800 \mathrm{mg}$ twice daily or placebo (1:1 for $200 \mathrm{mg}$ and 3:1 for other doses) for 5 days. The primary endpoint was time to SARS-CoV-2 RNA clearance ( $<1,018$ copies/mL), as measured by quantitative reverse transcriptase PCR of nasopharyngeal swabs. By day 28 (end of study), SARS-CoV-2 RNA clearance was seen in 91.3, 78.7 and $92.5 \%$ of patients in the molnupiravir 200, 400 and 800 mg groups, respectively, versus $80.3 \%$ in the placebo group. The median time to SARS-CoV-2 RNA clearance was significantly ( $p=0.013$ ) shorter for molnupiravir $800 \mathrm{mg}$ versus placebo (14.0 vs 15.0 days), with no significant between-group differences seen for other doses. Molnupiravir also dosedependently decreased infectious SARS-CoV-2. On day 5, the proportion of patients positive for infectious SARS-CoV-2 in nasopharyngeal swabs was significantly lower in the 400 $\mathrm{mg}(0 \%$ of $42 ; p=0.034)$ and $800 \mathrm{mg}(0 \%$ of $53 ; p=0.027)$ groups than in the placebo group (11.1\% of 54) [20].

Molnupiravir reduced the risk of hospitalization or death in patients with COVID-19 in a randomized, double-blind, placebo-controlled, global, phase II/III trial (MOVe-OUT; NCT04575597) [18, 37]. The trial predominantly (and exclusively in the phase III component) enrolled non-hospitalized adults with laboratory-confirmed mild to moderate COVID19 , at least one risk factor associated with poor disease outcomes and symptom onset within 5 days prior to randomization. Patients received molnupiravir 200, 400 or $800 \mathrm{mg}$ in the phase II component [18] and $800 \mathrm{mg}$ in the phase III component [37] or placebo twice daily for 5 days. The final dose selection for evaluation in the phase III component was based on the totality of clinical efficacy, virologic and safety outcomes across the molnupiravir clinical programme [36]. The primary endpoint of MOVe-OUT was the incidence of hospitalization for any cause or death through day 29 in the modified intent-to-treat population [18, 37]. In the phase II component ( $n=302), 3.1 \%$ of patients in the combined molnupiravir group were hospitalized or died, compared with $5.4 \%$ in the placebo group [18]. Secondary endpoints of time to progression and time to sustained resolution or improvement of COVID-19 symptoms also favoured molnupiravir over placebo. At the end of treatment (day 5), molnupiravir $800 \mathrm{mg}$ was associated with numerically higher rates of SARS-CoV-2 viral clearance versus placebo (12.7 vs $3.6 \%$ of patients). These results supported further evaluation of molnupiravir in the phase III component [18].

In a planned interim analysis of the phase III component of MOVe-OUT $(n=775)$, molnupiravir met the prespecified superiority criterion versus placebo; the primary endpoint incidence was 7.3 versus $14.1 \%$ (difference $6.8 \%$; 95\% CI -11.3 to $-2.4 ; p=0.001$ ) [37]. Due to these positive results, the trial was stopped early based on recommendation by an independent data monitoring committee. In the final analysis (i.e. all randomized population; $n=1433$ ), the corresponding incidence was 6.8 versus $9.7 \%$ (difference $3.0 \%$; $95 \%$ CI -5.9 to -0.1 ). In a prespecified supportive analysis of the final all randomized population, the incidence of COVID-19-related hospitalizations or death was $6.3 \%$ in the molnupiravir group versus $9.2 \%$ in the placebo group (difference $2.8 \%$; $95 \%$ CI -5.7 to 0.0 ). A time-to-event analysis showed that molnupiravir reduced the risk of hospitalization or death by $31 \%$ versus placebo. The incidence of 29 -day allcause mortality was $0.1 \%$ in the molnupiravir group and $1.3 \%$ in the placebo group, corresponding to an $89 \%$ reduction in the risk of death. In most prespecified subgroups (based on race, age, days since symptom onset, baseline disease severity, SARS-CoV-2 nucleocapsid antibody status and qualitative assay at baseline and risk factors for severe disease), the point estimates for the difference in the primary endpoint incidence favoured molnupiravir over placebo. Consistent treatment benefit in terms of the primary endpoint was seen in subgroups based on causative SARS-CoV-2 variants of concern (delta, mu, gamma) based on data available at the time of the report. Molnupiravir also provided other clinical benefits, as assessed by the WHO Clinical Progression Scale and patient-reported COVID-19 symptoms. In exploratory analyses, based on the available data, molnupiravir reduced mean SARS-CoV-2 nasopharyngeal RNA titers from baseline at days 3 and 5 versus placebo (adjusted relative reduction 42 and 53\%) [37].

\subsubsection{Hospitalized Patients}

Molnupiravir showed no clinical benefit in hospitalized adults with COVID-19 in a randomized, double-blind, placebo-controlled phase II trial (MOVe-IN; NCT04575584) [19]. Patients aged $\geq 18$ years requiring in-hospital treatment for laboratory-confirmed COVID-19 with symptom onset within 10 days were randomized to molnupiravir 200, 400 or $800 \mathrm{mg}$, or placebo twice daily for 5 days $(\mathrm{n}=304)$. The primary efficacy endpoint was sustained recovery rate, defined as the proportion of patients being alive and either not hospitalized or medically ready for hospital discharge. The sustained recovery rate was similar between molnupiravir and placebo groups (81.5-85.2\% vs $84.7 \%)$ and the median time to sustained recovery was 9 days in all groups. All-cause mortality rates were also similar between the treatment groups. The lack of clinical benefit with molnupiravir in the hospitalized population was thought to be related to delayed treatment initiation in relation to the temporal pattern of COVID-19 symptom onset and illness severity [19]. 
Key clinical trials of molnupiravir (Merck and/or Ridgeback biotherapeutics)

\begin{tabular}{|c|c|c|c|c|c|}
\hline Drug & Indication & Phase & Status & Location(s) & Identifier \\
\hline Molnupiravir & $\begin{array}{l}\text { COVID-19 } \\
\text { prevention }\end{array}$ & III & Recruiting & Multinational & $\begin{array}{l}\text { MOVe-AHEAD; NCT04939428; 4482-013; } \\
\text { EudraCT2021-000904-39; MK4482-013 }\end{array}$ \\
\hline Molnupiravir & $\begin{array}{l}\text { Non-hospitalized } \\
\text { adults with } \\
\text { COVID-19 }\end{array}$ & II/III & $\begin{array}{l}\text { Stopped early for } \\
\text { efficacy }\end{array}$ & Multinational & $\begin{array}{l}\text { MOVe-OUT; NCT04575597; } \\
\text { EudraCT2020-003368-24; MK-4482-002; } \\
\text { PHRR201209-003186; jRCT2031210148 }\end{array}$ \\
\hline Molnupiravir & $\begin{array}{l}\text { Hospitalized adults } \\
\text { with COVID-19 }\end{array}$ & II/III & $\begin{array}{l}\text { Discontinued after } \\
\text { phase II }\end{array}$ & Multinational & $\begin{array}{l}\text { MOVe-IN; NCT04575584; } \\
\text { EudraCT2020-003367-26; MK4482-001 }\end{array}$ \\
\hline Molnupiravir & $\begin{array}{l}\text { Hospitalized adults } \\
\text { with COVID-19 }\end{array}$ & II & Recruiting & USA & END-COVID; NCT04405739; EIDD-2801-2004 \\
\hline Molnupiravir & COVID-19 & II & Completed & USA & NCT04405570; EIDD-2801-2003 \\
\hline
\end{tabular}

\subsection{Adverse Events}

Molnupiravir was well tolerated in patients with COVID19 in clinical trials $[18,19,34,37]$. In a phase I trial (NCT04746183), outpatients with COVID-19 received molnupiravir 300-800 mg twice daily for 5 days plus standard-ofcare or standard-of-care alone (control) [34]. A molnupiravir dose was deemed unsafe if the probability of $\geq 30 \%$ excess dose-limiting toxicity over controls was $\geq 25 \%$. For the 800 $\mathrm{mg}$ dose, this probably was estimated at $0.9 \%$ [34].

In phase II trials, molnupiravir up to $800 \mathrm{mg}$ twice daily for 5 days was not associated with any dose-limiting adverse events (AEs), specific safety signals or clinically relevant abnormal laboratory test results (including haematological toxicity) in non-hospitalized [18] or hospitalized [19] patients with COVID-19. The AE profile of molnupiravir was generally comparable to that of placebo $[18,19]$.

In the phase III component of MOVe-OUT, molnupiravir did not differ significantly from placebo with respect to the incidence of overall and treatment-related AEs [37]. At least one treatment-related AE occurred in 57 of 710 (8.0\%) molnupiravir recipients and 59 of $701(8.4 \%)$ placebo recipients; the most common (incidence $\geq 1 \%$ ) of these were diarrhoea ( 1.7 vs $2.1 \%$ ), nausea ( 1.4 vs $0.7 \%$ ) and dizziness (1.0 vs. $0.7 \%$ ). No molnupiravir recipient and one placebo recipient experienced a treatment-related serious AE. Four molnupiravir and three placebo recipients discontinued study treatments because of treatment-related AEs. Two molnupiravir recipients and 12 placebo recipients died from AEs, none related to study treatment [37].

\subsection{Ongoing Clinical Trials}

The AGILE platform trial (NCT04746183) is still ongoing. The efficacy and safety of molnupiravir is being tested in a UK-based real-world study. A randomized, doubleblind, placebo-controlled, multinational phase III trial
(MOVe-AHEAD; NCT04939428) is evaluating molnupiravir for preventing the spread of COVID-19 within households. A randomized, double-blind, placebo-controlled, multicentre phase II trial (END-COVID; NCT04405739) is evaluating the efficacy and safety of molnupiravir in newly hospitalized adults with PCR-confirmed COVID-19.

\section{Current Status}

Molnupiravir received its first approval on 4 November 2021 in the UK for the treatment of mild-to-moderate COVID-19 in adults with a positive SARS-CoV-2 diagnostic test and who have at least one risk factor for developing severe illness [7]

Supplementary Information The online version contains supplementary material available at https://doi.org/10.1007/s40265-022-01684-5.

\section{Declarations}

Funding The preparation of this review was not supported by any external funding.

Authorship and conflict of interest During the peer review process the manufacturer of the agent under review was offered an opportunity to comment on the article. Changes resulting from any comments received were made by the authors on the basis of scientific completeness and accuracy. Yahiya Y. Syed is a salaried employee of Adis International Ltd/Springer Nature, and declares no relevant conflicts of interest. All authors contributed to the review and are responsible for the article content.

Ethics approval, Consent to participate, Consent to publish, Availability of data and material, Code availability Not applicable.

\section{References}

1. Painter GR, Natchus MG, Cohen O, et al. Developing a direct acting, orally available antiviral agent in a pandemic: the evolution of 
molnupiravir as a potential treatment for COVID-19. Curr Opin Virol. 2021;50:17-22.

2. Holman W, Holman W, McIntosh S, et al. Accelerated first-inhuman clinical trial of EIDD-2801/MK-4482 (molnupiravir), a ribonucleoside analog with potent antiviral activity against SARSCoV-2. Trials. 2021;22(1):561.

3. Hillen HS, Kokic G, Farnung L, et al. Structure of replicating SARS-CoV-2 polymerase. Nature. 2020;584(7819):154-6.

4. Amin SA, Jha T. Fight against novel coronavirus: a perspective of medicinal chemists. Eur J Med Chem. 2020;201:112559.

5. Vicenti I, Zazzi M, Saladini F. SARS-CoV-2 RNA-dependent RNA polymerase as a therapeutic target for COVID-19. Expert Opin Ther Pat. 2021;31(4):325-37.

6. Gilead Sciences. Veklury ${ }^{\circledR}$ (remdesivir) injection, for intravenous use: US prescribing information. 2020. https://www.vekluryhcp. com/. Accessed 14 Jan 2022.

7. Merck Sharp \& Dohme (UK) Limited. Lagevrio $200 \mathrm{mg}$ hard capsules: UK prescribing information. 2021. https://products.mhra. gov.uk/. Accessed 14 Jan 2022.

8. UK Medicines and Healthcare products Regulatory Agency. First oral antiviral for COVID-19, Lagevrio (molnupiravir), approved by MHRA [media release]. 4 Nov 2021. https://www.gov.uk.

9. Merck \& Co. Merck and Ridgeback Bio announce closing of collaboration and licensing transaction [media release]. 1 Jul 2020. http://www.merck.com.

10. Merck \& Co, Ridgeback Biotherapeutics. Merck and Ridgeback Bio collaborate to advance development of novel antiviral candidate, EIDD-2801 [media release]. 26 May 2020. http://www. merck.com.

11. Ridgeback Biotherapeutics. Ridgeback Biotherapeutics and Drug Innovations Ventures at Emory partner to develop clinical stage coronavirus treatment [media release]. 19 Mar 2020. http://www. ridgebackcap.com.

12. Cipla. Pharma majors collaborate for clinical trial of investigational oral anti-viral drug molnupiravir for COVID-19 [media release]. 29 Jun 2021. http://www.cipla.com.

13. Medicines Patent Pool. The Medicines Patent Pool (MPP) and Merck enter into license agreement for molnupiravir, an investigational oral antiviral COVID-19 medicine, to increase broad access in low- and middle-income countries [media release]. 27 Oct 2021. http://www.medicinespatentpool.com.

14. Merck \& Co. Merck and Ridgeback announce supply agreement with UNICEF for molnupiravir, an investigational oral antiviral COVID-19 medicine [media release]. 2022. https://www.merck. $\mathrm{com} /$.

15. Painter WP, Holman W, Bush JA, et al. Human safety, tolerability, and pharmacokinetics of molnupiravir, a novel broad-spectrum oral antiviral agent with activity against SARS-CoV-2. Antimicrob Agents Chemother. 2021. https://doi.org/10.1128/aac. 02428-20.

16. Lee CC, Hsieh CC, Ko WC. Molnupiravir-a novel oral antiSARS-CoV-2 agent. Antibiotics. 2021. https://doi.org/10.3390/ antibiotics 10111294.

17. McIntosh JA, Benkovics T, Silverman SM, et al. Engineered ribosyl-1-kinase enables concise synthesis of molnupiravir, an antiviral for COVID-19. ACS Cent Sci. 2021;7(12):1980-5.

18. Caraco Y, Crofoot GE, Moncada PA, et al. Phase 2/3 trial of molnupiravir for treatment of Covid-19 in nonhospitalized adults. NEJM Evid. 2021. https://doi.org/10.1056/EVIDoa2100043.

19. Arribas JR, Bhagani S, Lobo SM, et al. Randomized trial of molnupiravir or placebo in patients hospitalized with Covid-19. NEJM Evid. 2021. https://doi.org/10.1056/EVIDoa2100044.

20. Fischer WA, Eron JJ Jr, Holman W, et al. A phase 2a clinical trial of molnupiravir in patients with COVID-19 shows accelerated
SARS-CoV-2 RNA clearance and elimination of infectious virus. Sci Transl Med. 2022. https://doi.org/10.1126/scitranslmed.abl74 30.

21. Vangeel L, Chiu W, De Jonghe S, et al. Remdesivir, molnupiravir and nirmatrelvir remain active against SARS-CoV-2 omicron and other variants of concern. Antiviral Res. 2022. https://doi.org/10. 1016/j.antiviral.2022.105252.

22. Li P, Wang $\mathrm{Y}$, Lavrijsen $\mathrm{M}$, et al. SARS-CoV-2 omicron variant is highly sensitive to molnupiravir, nirmatrelvir, and the combination. Cell Res. 2022. https://doi.org/10.1038/ s41422-022-00618-w.

23. Bojkova D, Widera M, Ciesek S, et al. Reduced interferon antagonism but similar drug sensitivity in omicron variant compared to delta variant of SARS-CoV-2 isolates. Cell Res. 2022. https://doi. org/10.1038/s41422-022-00619-9.

24. Takashita E, Kinoshita N, Yamayoshi S, et al. Efficacy of antibodies and antiviral drugs against Covid-19 omicron variant. N Engl J Med. 2022. https://doi.org/10.1056/NEJMc2119407.

25. Dabrowska A, Szczepanski A, Botwina P, et al. Efficacy of antiviral drugs against the omicron variant of SARS-CoV-2. bioRxiv. 2021. https://doi.org/10.1101/2021.12.21.473268.

26. Rosales R, McGovern BL, Rodriguez ML, et al. Nirmatrelvir, molnupiravir, and remdesivir maintain potent in vitro activity against the SARS-CoV-2 omicron variant. bioRxiv. 2022. https:// doi.org/10.1101/2022.01.17.476685.

27. Wahl A, Gralinski LE, Johnson CE, et al. SARS-CoV-2 infection is effectively treated and prevented by EIDD-2801. Nature. 2021;591(7850):451-7.

28. Cox RM, Wolf JD, Plemper RK. Therapeutically administered ribonucleoside analogue MK-4482/EIDD-2801 blocks SARSCoV-2 transmission in ferrets. Nat Microbiol. 2021;6(1):11-8.

29. Sheahan TP, Sims AC, Zhou S, et al. An orally bioavailable broadspectrum antiviral inhibits SARS-CoV-2 in human airway epithelial cell cultures and multiple coronaviruses in mice. Sci Transl Med. 2020. https://doi.org/10.1126/scitranslmed.abb5883.

30. Agostini ML, Pruijssers AJ, Chappell JD, et al. Small-molecule antiviral beta-D-N(4)-hydroxycytidine inhibits a proofreadingintact coronavirus with a high genetic barrier to resistance. J Virol. 2019. https://doi.org/10.1128/JVI.01348-19.

31. Gordon CJ, Tchesnokov EP, Schinazi RF, et al. Molnupiravir promotes SARS-CoV-2 mutagenesis via the RNA template. J Biol Chem. 2021. https://doi.org/10.1016/j.jbc.2021.100770.

32. Kabinger F, Stiller C, Schmitzová J, et al. Mechanism of molnupiravir-induced SARS-CoV-2 mutagenesis. Nat Struct Mol Biol. 2021;28(9):740-6.

33. Troth S, Butterton J, DeAnda CS, et al. Letter to the Editor in response to Zhou et al. J Infect Dis. 2021;224(8):1442-3.

34. Khoo SH, Fitzgerald R, Fletcher T, et al. Optimal dose and safety of molnupiravir in patients with early SARS-CoV-2: a phase I, open-label, dose-escalating, randomized controlled study. J Antimicrob Chemother. 2021;76(12):3286-95.

35. Gao W, Bihorel S, Humphrey R, et al. Population pharmacokinetics analysis for molnupiravir in adults with COVID-19 [abstract no. PIIIB-011]. In: American Conference on Pharmacometrics. 2021.

36. Chawla A, Cao Y, Stone J, et al. Model-based dose selection for the phase 3 evaluation of molnupiravir (MOV) in the treatment of COVID-19 in adults [abstract no. 04736]. In: 31st European Congress of Clinical Microbiology and Infectious Diseases. 2021.

37. Jayk Bernal A, Gomes da Silva MM, Musungaie DB, et al. Molnupiravir for oral treatment of Covid-19 in nonhospitalized patients. N Engl J Med. 2021. https://doi.org/10.1056/NEJMo a2116044. 